\title{
Effect of Crystallization of the Grain-Boundary Phase on the Thermal Diffusivity of a Sialon Ceramic
}

\author{
LARRY D. BentSen* AND D. P. H. HASSElman* \\ Department of Materials Engineering, Virginia Polytechnic Institute and State University, \\ Blacksburg, Virginia 24061

\section{TSENG-YING TIEN*} \\ Materials and Metallurgical Engineering, The University of Michigan, Ann Arbor, \\ Michigan 48109
}

Crystallization of the glassy grain-boundary phase in a series of sialon ceramics fabricated using a range of hot-pressing schedules increased the thermal diffusivity at room temperature by an average of $\approx 10 \%$. For samples made by a given hotpressing schedule, the relative difference in thermal diffusivity between composites containing a glassy grain-boundary phase and those in which this phase had crystallized decreased with increasing temperature. This behavior is attributed to enhanced phonon scattering in the crystalline grain-boundary phase.

The DEnsification during sintering of 1 polycrystalline ceramics for highperformance applications can be enhanced significantly by the addition of a sintering aid. This is particularly so for $\mathrm{Si}_{3} \mathrm{~N}_{4}$-based ceramics, which do not densify readily without the assistance of additives. For $\mathrm{Si}_{3} \mathrm{~N}_{4}$, additives commonly consist of the oxides of magnesium, yttrium, or aluminum. At the fabrication temperatures, the presence of these additives results in the formation of a liquid phase at the grain boundaries. This liquid phase can act either as a lubricant to promote particle transport or as a medium for material transport by solution and reprecipitation of the matrix phase. On cooling, the liquid at the grain boundaries and triple points becomes a glass.

The presence of this grain-boundary

CONTRIBUTING EDITOR-H.K. BOWEN

Presented at the Fall Meeting, The American Ceramic Society, Columbus, OH, October 31, 1983 (Basic Science Division No. 17-B-83F). Received (Basic Science Division No. 17-B-83F), Received
November 7 . 1983; revised copy received February 10, 1984; approved February 10, 1984.

Supported in part by the U.S. Department of Energy under Contract No. DE-AC02-80ER 10619 and by the Office of Naval Research under Contract No. N00014-78-C-0431.

"Member, the American Ceramic Society.
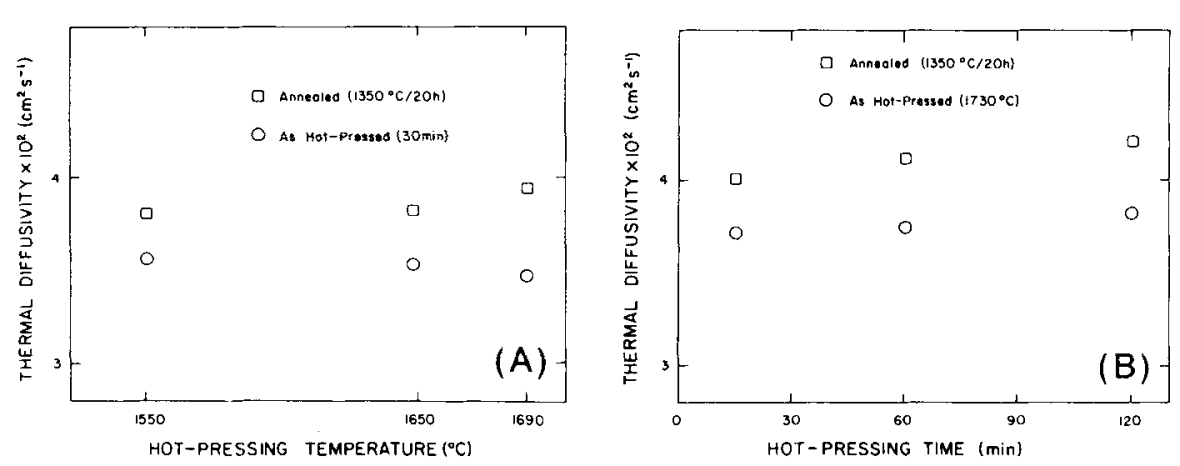

Fig. 1. Effect of annealing at $1350^{\circ} \mathrm{C}$ for $20 \mathrm{~h}$ on room-temperature thermal diffusivity of $\beta$-sialon/garnet $(A)$ hot-pressed at varied temperatures for $30 \mathrm{~min}$ and $(B)$ hot-pressed at $1730^{\circ} \mathrm{C}$ for varied times. phase, which can vary from just a few percent to over $10 \%$, is expected to have an effect on the continuum properties of the final densified product. This effect is well known for mechanical behavior, especially at temperatures sufficiently high that the grain-boundary phase can undergo significant viscous deformation.

The effect of the grain-boundary phase on the continuum properties of polycrystalline aggregates also is expected to be a function of the degree of crystallinity. In terms of the objectives of this study, note that glassy (amorphous) materials generally exhibit a much lower thermal conductivity and diffusivity than their crystalline counterparts of identical composition ${ }^{1.2}$ because of enhanced phonon scattering. A polycrystalline ceramic with a grain-boundary phase can be considered to be a composite. Cheng and Vachon ${ }^{3}$ reported that the thermal conductivity of composites is a function of the thermal conductivity, volume fraction, and distribution of each component. Continuous phases are particularly effective in affecting the thermal conductivity of composites. For this reason, the presence of a grain-boundary phase and the degree of its crystallinity are expected to have a significant effect on the thermal transport properties of polycrystalline aggregates. diffusivity of $\beta$-sialon/garnet hot-pressed at $1690^{\circ} \mathrm{C}$ for 30 min compared to that of $\beta$-sialon garnet hot-pressed under same conditions and then annealed at $1350^{\circ} \mathrm{C}$ for $20 \mathrm{~h}$. Curves represent linear least-squares fits of measured thermal diffusivity to reciprocal absolute temperature. 
nace with a protective nitrogen atmosphere The direction of heat flow in the specimens was parallel to the hot-pressing direction.

\section{RESULTS AND DISCUSSION}

Figure $1(A)$ compares the data for the thermal diffusivity at room temperature before and after the annealing of samples hotpressed for $30 \mathrm{~min}$ at varied temperatures. Similarly, Fig. $1(B)$ compares the corresponding data for specimens hot-pressed at $1730^{\circ} \mathrm{C}$ for varied time periods. Both sets of data show an $\approx 10 \%$ increase in the thermal diffusivity after annealing, regardless of the hot-pressing conditions.

Figure 2 compares the temperature dependence of the thermal diffusivity for sialon samples with a glassy and a crystalline grain-boundary phase. Both samples were hot-pressed for $30 \mathrm{~min}$ at $1690^{\circ} \mathrm{C}$. The relative difference in the thermal diffusivity decreases with incraasing temperature. This decrease is $\mathrm{ex}_{\mathrm{r}}$ ' since enhanced phonon scattering generally produces a greater relative decrease in the thermal conductivity and diffusivity of crystalline materials than for glassy materials. The data in Fig. 2 were obtained up to $1000^{\circ} \mathrm{C}$ to avoid crystallization of the grain-boundary phase at higher temperatures. Such crystallization would lead to a permanent increase in the thermal diffusivity of the "as-hot-pressed" sample if measurements had been conducted to temperatures as high as $1200^{\circ}$ or $1300^{\circ} \mathrm{C}$. Although the temperature dependence of the thermal diffusivity was measured for only one hot-pressing condition, it is anticipated that the samples for the other hot-pressing conditions would exhibit a behavior similar to that shown in Fig. 2 .

\section{REFERENCES}

'W. D. Kingery, H. K. Bowen, and D. R. Uhlmann, Introduction to Ceramics, $2 d$ ed., Wiley, New York. 1976.

${ }^{2}$ K. Chyung, G. E. Youngblood, and D.P. H. Hasselman, "Effect of Crystallization on the Therma] Diffusivity of a Cordierite Glass-Ceramic," J. Am. Ceram. Soc. 61 [11-12] 530-31 (1978).

${ }^{3}$ S. C. Cheng and R. I. Vachon, "The Prediction of the Thermal Conductivity of Two- and Three-Phase of the Thermal Conductivity of Two- and Three-Phase Solid Heterogeneous Mixtures
Transfer, 12 [3] 249-64 (1969).

IT. Y. Tien, "Effect of Crystallization of Grain Boundary Phase on the High Temperature Strength of Silicon Nitride Ceramics," Interim Tech. Rept. to DOE, November 1982.

${ }^{5}$ W. J. Parker, R. J. Jenkins, C. P. Butler, and G. L. Abbott, "Flash Method of Determining Thermal Diffusivity, Heat Capacity, and Thermal Conductivity," Diffusivity, Heat Capacity, and Thermal Co
J. Appl. Phys., 32 [9] 1679-84 (1961).

\section{Cation Valence in $\mathrm{VO}_{x}$}

\author{
Robert L. Hsieh, * Jerome B. Cohen, ${ }^{*}$ and Panayotis Georgopoulos \\ Department of Materials Science and Engineering, The Technological Institute, Northwestern \\ University, Evanston, Illinois 60201
}

By comparing the position of the $\mathrm{K}$ absorption edge of $\mathrm{VO} \mathrm{O}_{x}$ to that of $\mathrm{V}$ metal, $\mathrm{V}_{2} \mathrm{O}_{4}$, and $\mathrm{V}_{2} \mathrm{O}_{5}$, it is concluded that the cation in $V \mathrm{O}_{x}$ has the classical valence of $2+$ only for oxygen-rich compositions.

$\mathbf{T}_{\mathrm{k} \in \mathrm{s}}^{\mathrm{H}}$ HE CONCEPT of classical valence plays a key role in the defect chemistry of nonstoichiometric oxides. Yet many of these materials are semiconductors or metallic in their behavior.

The oxide $\mathrm{VO}_{x}(0.75<x<1.3)$ is one such example; it is metallic for vanadiumrich compositions and a semiconductor for oxygen-rich material.' Theoretical calculations ${ }^{2-4}$ and experiments involving combined $\mathrm{X}$-ray diffraction and highvoltage electron microscopy (critical voltage and disappearing Kikuchi lines ${ }^{5,6}$ ) suggest that the valence is less than the classical value of $2+$. Furthermore, although it is assumed generally that vacant cations produce a higher valence on some of the remaining cations, in at least one carbide, charge compensation appears to occur by a decrease in the anion valence. ${ }^{7}$ The purpose of this communication is to probe the cation valence in $\mathrm{VO}_{x}$ (at three compositions) by examining the chemical shift of the $\mathrm{K}$ absorption edge of vanadium.

CONTRIBUTING EDITOR - G. LEWIS

\footnotetext{
Received December 14, 1983; approved January

Research is a portion of a senior thesis by R. Hsieh at Northwestern University.

Supported in part by the National Science Foundation under Contract No. DMR-82-16972.

"Member, the American Ceramic Society.

*Now with the Materials Science Dept., Stanford
} 31, 1984 . University, Stanford, CA

\section{Experimental Procedure}

A description of the preparation of the $\mathrm{VO}_{x}$ samples is given in Ref. 6. Crystals were ground to a powder and coated on adhesive tape. Three such tapes were superimposed to obtain suitable $\mathrm{X}$-ray absorption and mounted on a cardboard frame. Similar samples were prepared from high-purity $\mathrm{V}_{2} \mathrm{O}_{4}, \mathrm{~V}_{2} \mathrm{O}_{5}$, and annealed filings of vanadium metal.

Near-edge spectra, shown in Fig. 1 , were obtained using a $12-\mathrm{kW}$ rotating anode generator ${ }^{\ddagger}$ with Mo target operating at $30 \mathrm{kV}, 160 \mathrm{~mA}$. The energy of the radiation was selected by the 220 reflection from a thin Si slice bent elastically to focus the radiation at a slit used to improve the energy resolution. The bending jig was mounted on a diffractometer ${ }^{\ddagger}$ in a helium atmosphere. A sample was placed downstream from the slit, and the beam intensity was measured (simultaneously) before and after it entered the sample with gas-flow detectors. The incident-beam detector was smaller than that for the transmitted beam, and the gas pressure was adjusted to absorb only $10 \%$ of the beam. The output of this detector was used in a feedback circuit to maintain a constant intensity incident on the sample for all energies. (A more complete description of the apparatus is given in Ref. 8.) A 100-eV range around the vanadium metal absorption edge was

${ }^{\dagger}$ Rigaku Denki Corp., Tokyo, Japan.

${ }^{\dagger}$ Picker Corp., Cleveland, OH. sampled for $30 \mathrm{~s}$ at $0.5-\mathrm{eV}$ intervals. The range from 5430 to $5730 \mathrm{eV}$ was recorded at 5-eV steps, each for $10 \mathrm{~s}$. The absorption-edge jump height was obtained by curve-fitting the pre- and post-edge portions of the extended spectra, and the detailed spectra for all samples were normalized such that the absorption jump equals unity. The position of the edge was found from the derivative of the (normalized) data, ignoring any pre-edge peaks. Data acquisition and processing were controlled by a microcomputer system. ${ }^{\S}$

\section{RESULTS AND DISCUSSION}

In Fig. 2, the normalized absorption coefficient and its derivative are shown for $\mathrm{V}$ metal. The edge is at $2.72 \mathrm{eV}$ from (the arbitrary) zero; the first maximum in the derivative is a pre-edge peak. The shifts were correlated with the coordination charge $(C C)$ because previous work ${ }^{9-11}$ has indicated a linear relation. Coordination charge is defined as:

$C C=m I$

where $m$ is the valence and $I$ the ionicity calculated from the Pauling formula ${ }^{12}$ :

$I=1-\exp \left[-(1 / 4)\left(X_{\mathrm{V}}-X_{\mathrm{O}}\right)^{2}\right]$

where $X_{\mathrm{V}}$ and $X_{\mathrm{O}}$ are $\mathrm{V}$ and $\mathrm{O}$ electronegativities (having values of 1.63 and 3.44 , respectively ${ }^{13}$ ). The results are shown in Fig. 3 in which the straight line is drawn through the points for $\mathrm{V}, \mathrm{V}_{2} \mathrm{O}_{4}$, and $\mathrm{V}_{2} \mathrm{O}_{5}$. The coordination charges were obtained from the measured edge shifts for $\mathrm{VO}_{0.80}$, $\mathrm{VO}_{1.02}$, and $\mathrm{VO}_{1.25}$ in Fig. 3, and valence results (Table $\mathrm{I}$ ) were determined using Eqs. (1) and (2). The quoted errors arise from the uncertainty in the straight line fit in Fig. 3 and from uncertainties in locating the peaks in the derivative spectra.

It is clear that in $\mathrm{VO}_{x}$, the valence only approaches the classical value of $2+$ in the most oxygen-rich compositions, in agree-

$\$ 8080$, Intel Corp., Santa Clara, CA. 